\title{
Wide-Field Megahertz OCT Imaging of Patients with Diabetic Retinopathy
}

\author{
Lukas Reznicek, ${ }^{1}$ Jan P. Kolb, ${ }^{2,3}$ Thomas Klein, ${ }^{2}$ Kathrin J. Mohler, ${ }^{2}$ Wolfgang Wieser, ${ }^{2}$ \\ Robert Huber, ${ }^{3}$ Marcus Kernt, ${ }^{4}$ Josef Märtz, ${ }^{4}$ and Aljoscha S. Neubauer ${ }^{4}$ \\ ${ }^{1}$ Department of Ophthalmology, Technical University of Munich, Ismaninger Strasse 22, 81675 Munich, Germany \\ ${ }^{2}$ Institute for Biomolecular Optics, Ludwig Maximilians University, Oettingenstrasse 67, 80538 Munich, Germany \\ ${ }^{3}$ Institute of Biomedical Optics, University of Lübeck, Peter-Monnik-Weg 4, 23562 Lübeck, Germany \\ ${ }^{4}$ Department of Ophthalmology, Ludwig Maximilians University, Mathildenstrasse 8, 80336 Munich, Germany
}

Correspondence should be addressed to Lukas Reznicek; lukas.reznicek@mri.tum.de

Received 31 March 2015; Accepted 30 June 2015

Academic Editor: Subrata Chakrabarti

Copyright (C) 2015 Lukas Reznicek et al. This is an open access article distributed under the Creative Commons Attribution License, which permits unrestricted use, distribution, and reproduction in any medium, provided the original work is properly cited.

\begin{abstract}
Purpose. To evaluate the feasibility of wide-field Megahertz (MHz) OCT imaging in patients with diabetic retinopathy. Methods. A consecutive series of 15 eyes of 15 patients with diagnosed diabetic retinopathy were included. All patients underwent Megahertz OCT imaging, a close clinical examination, slit lamp biomicroscopy, and funduscopic evaluation. To acquire densely sampled, widefield volumetric datasets, an ophthalmic $1050 \mathrm{~nm}$ OCT prototype system based on a Fourier-domain mode-locked (FDML) laser source with $1.68 \mathrm{MHz}$ A-scan rate was employed. Results. We were able to obtain OCT volume scans from all included 15 patients. Acquisition time was 1.8 seconds. Obtained volume datasets consisted of $2088 \times 1044 \mathrm{~A}$-scans of $60^{\circ}$ of view. Thus, reconstructed en face images had a resolution of 34.8 pixels per degree in $x$-axis and 17.4 pixels per degree. Due to the densely sampled OCT volume dataset, postprocessed customized cross-sectional B-frames through pathologic changes such as an individual microaneurysm or a retinal neovascularization could be imaged. Conclusions. Wide-field Megahertz OCT is feasible to successfully image patients with diabetic retinopathy at high scanning rates and a wide angle of view, providing information in all three axes. The Megahertz OCT is a useful tool to screen diabetic patients for diabetic retinopathy.
\end{abstract}

\section{Introduction}

Diabetic retinopathy is a leading cause for blindness in industrialized countries [1]. The prevalence of diabetes and diabetic retinopathy is increasing worldwide including first world countries [2]. Thus, early detection of diabetic changes of the posterior ocular fundus for an individualized and optimized treatment is of high importance.

Multiple noninvasive diagnostic imaging tools for screening as well as detection and monitoring of the diabetic retinopathy are available, for example, fundus cameras $[3,4]$ or scanning laser ophthalmoscopy [5] for en face images of the posterior fundus or OCT scans for cross-sectional images of the central retina [6-8].

In general, retinal diseases such as diabetic retinopathy can manifest in the central as well as peripheral retina and can initially develop subtle pathologic alterations. The NHS Diabetic Eye Screening Program in the United Kingdom as well as the conclusions of Early Treatment Diabetic Retinopathy Study, for example, demands a field of view of $45^{\circ} \times$ $40^{\circ}$ for fundus cameras to ensure high sensitivity and specificity to successfully screen for diabetic retinopathy. Therefore, the requirements for valid images to effectively depict the posterior fundus are a wide angle of view and a high resolution. Fundus cameras fulfilling those requirements are able to obtain en face images in $x-y$-axis with useful information depicting potential diabetic pathologies located on the surface of the retina. On the other hand, commercially available OCT devices have been able to obtain crosssectional scans of the central retina but were limited to a narrow angle of view $\left(30 \times 20^{\circ}\right)$ and slow acquisition speed, 
thus failing to provide enough pixels per degree in reconstructed en face display modes.

The recently introduced ultrahigh-speed swept source Fourier-domain mode-locked (FDML) OCT [9-11] is feasible at high scanning rates providing images with a wide angle of view. The obtained volume scans of the retina allowed a reconstruction of high resolution en face images as well as histology-like cross-sectional retinal scans (B-frames) depicting the fundus beyond the central macular area with an image quality comparable to current established OCT devices [9].

The purpose of this study was to evaluate the feasibility of this novel device imaging diabetic patients with diagnosed diabetic retinopathy.

\section{Patients and Methods}

We retrospectively selected 15 eyes of 15 consecutive patients with diagnosed diabetic retinopathy (5 eyes with proliferative diabetic retinopathy versus 10 eyes with nonproliferative diabetic retinopathy) who were imaged between October 2013 and April 2014 in the MHz OCT study at the LMU Eye Hospital Munich (German Clinical Trial Register ID DRKS00005173). Three were females and 12 were males. Mean age was $57.4 \pm 11.1$ years (range $41-81$ years). Ethics committee approval was obtained in June 2013. Written informed consent was obtained from all patients prior to OCT imaging. The research adhered to the tenets of the Declaration of Helsinki.

All patients underwent Megahertz OCT imaging, a close clinical examination including measurements of refraction, best corrected VA, slit lamp biomicroscopy, and funduscopic evaluation. Patients were not dilated specifically for the imaging procedures. Only one eye per subject was selected to avoid any intraindividual correlations.

The ophthalmic OCT prototype system is based on a Fourier-domain mode-locked (FDML) laser source, which makes the acquisition of densely sampled, wide-field volumetric datasets possible. Details of this custom FDML MHz OCT system can be found in $[9,12,13]$.

In summary, the system employs a rapidly wavelengthtunable FDML laser with a center wavelength of $1050 \mathrm{~nm}$ for higher tissue penetration in contrast to spectral-domain OCT. It can obtain an ultrahigh number of 1.68 Mio depth scans (A-scans) per second. The sweep bandwidth of approximately $70 \mathrm{~nm}$ makes an axial resolution of $10 \mu \mathrm{m}$ in tissue possible. $1.6 \mathrm{~mW}$ sample arm power is incident on the eye, which is below the limits declared in the American National Standard Institute (ANSI) standards for safe ocular exposure (ANSI, "Safe Use of Lasers \& Safe Use of Optical Fiber Communications," American National Standard Institute, Z136 Committee). At this power, the device achieves a sensitivity of $91 \mathrm{~dB}$.

\section{Results}

We were able to obtain OCT volume scans from all included 15 patients. Acquisition time was 1.8 seconds. Twodimensional en face images were reconstructed by taking the average of each A-scan of the three-dimensional OCT

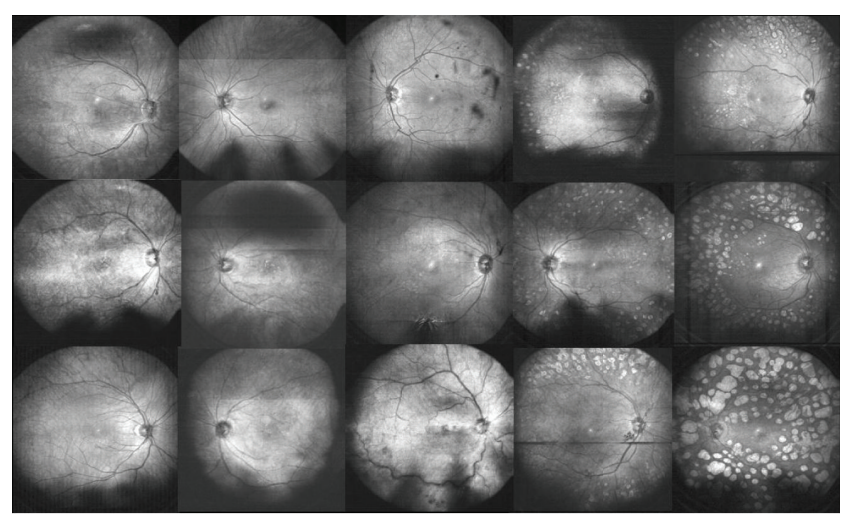

FIGURE 1: Reconstructed en face wide-field fundus images of all 15 patients; artifacts are shown in detail in Figure 2.

imaging data to provide a first overview. Then, arbitrary cross-sectional views can be obtained at every location for a closer examination as shown later on. Wide-field en face images from all patients can be seen in Figure 1.

Artifacts and possible quality modulations were pupillary or ciliary shadowing as known from other wide-field devices; motion artifacts such as eye blinks result in horizontal black stripes while saccades result in a "misalignment phenomenon" of the reconstructed en face images; see Figure 2. Ciliary shadowing is the only observed artifact that can be solved with pupil dilation.

Angle of view of the OCT imaging data was $60^{\circ}$ in diameter including the macular area, optic disc, and midperipheral retina significantly beyond the major vessel arcades; see Figure 3. For example, the NHS Diabetic Eye Screening Program in the United Kingdom demands a field of view of $45^{\circ} \times 40^{\circ}$ for fundus cameras, which is also covered by the three-dimensional data of the Megahertz OCT.

Obtained volume datasets consisted of $2088 \times 1044$ Ascans with $60^{\circ}$ field of view. Thus, reconstructed en face images had a resolution of 34.8 pixels per degree in $x$-axis and 17.4 pixels per degree in $y$-axis revealing fine details such as retinal microaneurysms (see Figures 4(a) and 4(c)). Figure 4(e) shows a cross-sectional scan of the imaged retina through a microaneurysm providing information in $z$-axis. Figures 4(b) and 4(d) display images of the same eye obtained with a wide-field scanning laser ophthalmoscope (Optos plc, Dunfermline, UK) serving as a reference.

Additionally, customized postprocessed B-frames depict retinal or choroidal regions of interest in $x-y$ - and $z$-axis; see Figure 5.

Pathologic changes such as an individual microaneurysm or a retinal neovascularisation could be imaged in arbitrary customized cross-sections extracted from to the densely sampled OCT volume dataset.

\section{Discussion}

In this study, for the first time, we were able to obtain images of the posterior fundus of patients with diabetic retinopathy applying the recently [9] novel wide-field Megahertz OCT 


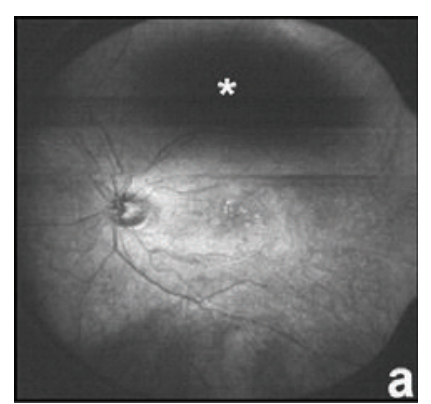

(a)

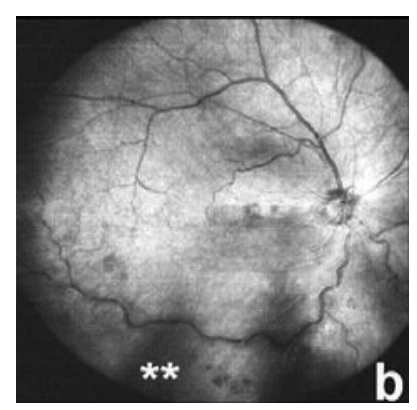

(b)

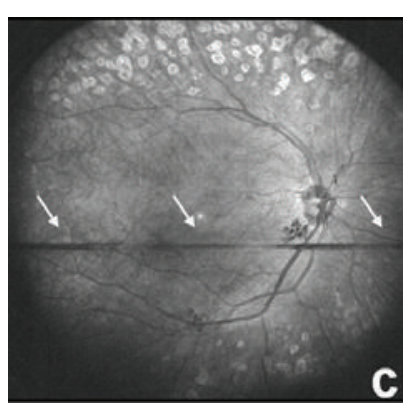

(c)

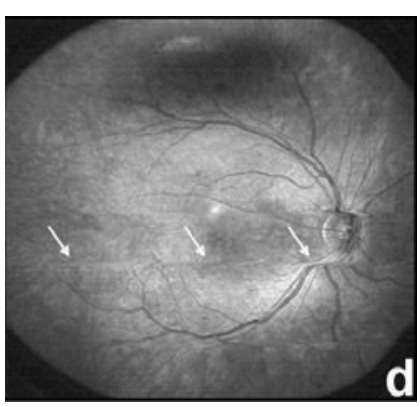

(d)

FiguRE 2: Artifacts of reconstructed en face wide-field fundus images with pupillary shadowing in (a) (*) and ciliary shadowing in (b) (**). Eye blinks result in horizontal black stripes seen in (c) and saccades in misalignments (see (d)) in the reconstructed en face images.

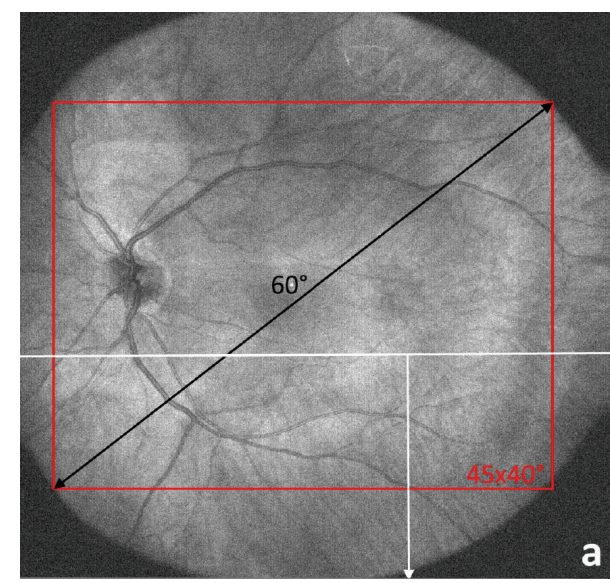

(a)

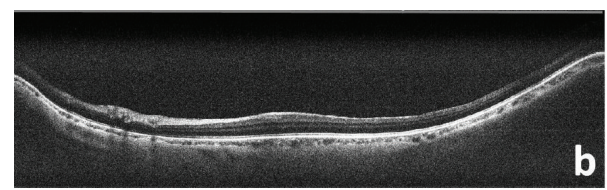

(b)

FIGURE 3: OCT images cover a field of view with a diameter of $60^{\circ}$ which corresponds to $45^{\circ} \times 40^{\circ}$ as shown in the en face reconstruction in (a). Cross-sectional views (B-frames) can be obtained at every location as seen in (b).

device. Volume scans from all imaged patients allowed for reconstructed high resolution en face images of the central and midperipheral fundus as well as cross-sectional scans of the retinal area of interest. A wide field of view is important for diagnostic tools used for retinal diseases to depict alterations located beyond the central posterior fundus, such as in the presented case series of patients with diabetic retinopathy.

Given the acquisition speed of $1.68 \mathrm{MHz}(1.680 .000 \mathrm{~A}$ scans per second), the obtained volume scans are densely sampled, provide a sufficient angle of view of the central as well as midperipheral fundus, and result in high resolution images of each customized postprocessed arbitrary B-scan extracted from the volume scan.

Commercially available noninvasive wide-field fundus devices are able to successfully depict the posterior fundus of patients with diabetic retinopathy in $x$ - $y$-axis [14] but cannot provide any information in $z$-axis. This means that devices imaging en face posterior fundus are not able to depict potential pathologies that do not show at the surface of the retina. The obtained $\mathrm{MHz}$ OCT volume scans of the retina on the other side allow the reconstruction of en face images which have been shown to exhibit comparable image quality to commercially available wide-field fundus devices [9] and have the additional significant advantage to provide information of the depicted area with cross-sectional scans also in $z$-axis. Cross-sectional images of patients with diabetic retinopathy obtained with the $\mathrm{MHz}$ OCT are therefore able to depict pathomorphologic alterations such as microaneurysms, neovascularizations (Figures 4 and 5), or intraretinal fluid and can identify their extension within middle or outer retinal layers. This additional information is missing in fundus photography due to the information provided only in $x-y$-axis.

The challenges with this new technology are the above mentioned ciliary and papillary shadowing which is known from other wide-field devices and motion artifacts (eye blinks, saccades) due to an acquisition time of 1.8 seconds, which in future could be reduced with faster beam scanners. Given the known inverse relationship between acquisition speed and obtained sensitivity, the achieved signal intensity of the acquired images can be significantly improved by averaging processes feasible at scanning rates beyond 1.000.000 A-scans per second and by applying higher optical power.

Limitations of our pilot study were the small number of 15 patients which was also limited by our ethics committee because of the pilot character of our study design. Further 


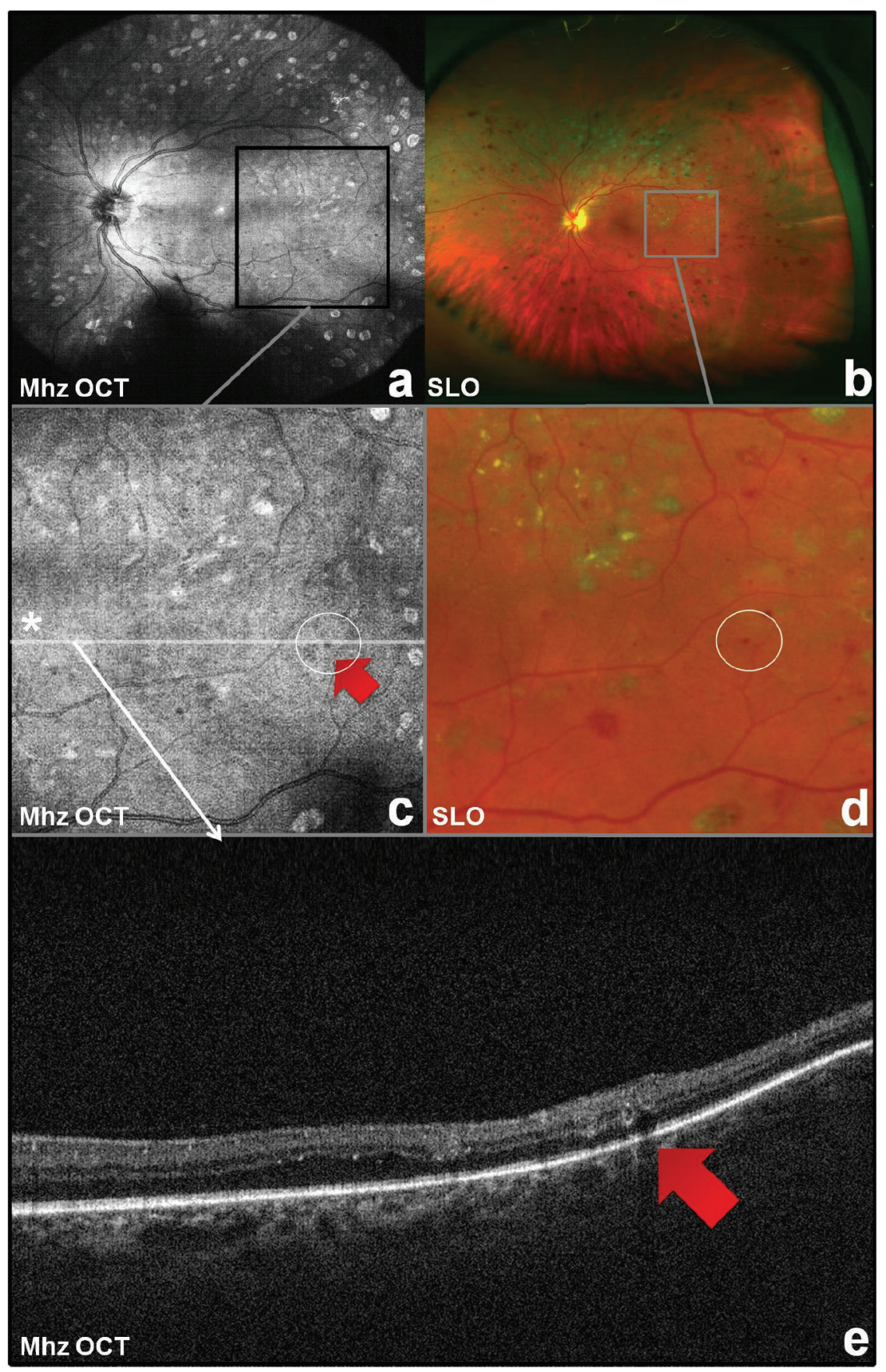

Figure 4: Reconstructed $\mathrm{MHz}$ OCT en face wide-field fundus image of left eye (a) with detail (c) versus wide-field scanning laser ophthalmoscopy (SLO) image (b) with detail (d). Cross-sectional MHz OCT scan from the same dataset (a, c) through a microaneurysm (red arrow) providing information in $z$-axis (e).

studies with large number of patients will have to confirm the feasibility. A further development of the presented $\mathrm{MHz}$ OCT device on the other hand will facilitate a higher competition with other diagnostic tools such as fundus cameras for screening purposes which require diagnostic tools that are easy to handle and inexpensive and create data volumes that can be transferred easily within an established network.

In summary, we were able to demonstrate the feasibility of the wide-field Megahertz OCT to successfully image patients with diabetic retinopathy at high scanning rates and a wide angle of view, providing information in all three axes. The Megahertz OCT is a useful tool to screen diabetic patients for diabetic retinopathy.

\section{Conflict of Interests}

The authors do not have any commercial interest in any of the materials and methods used in this study. 


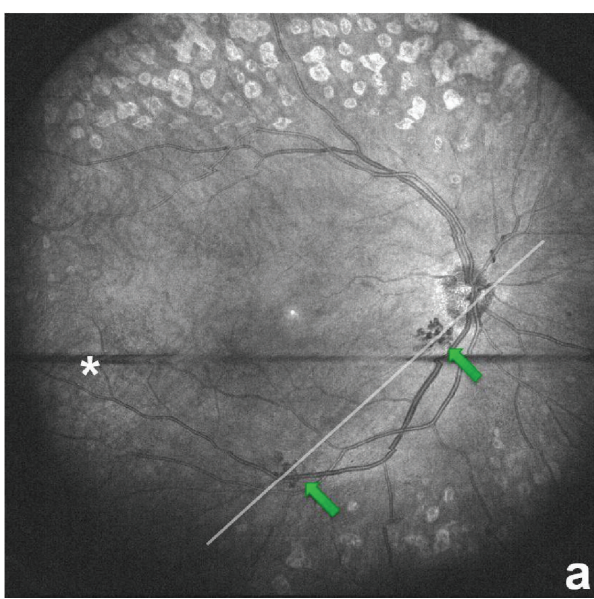

(a)

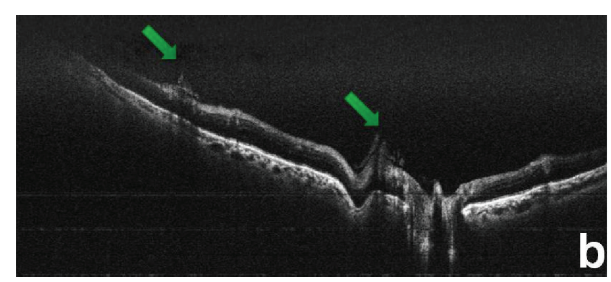

(b)

Figure 5: Reconstructed MHz OCT en face wide-field fundus image of right eye (a) with neovascularisations (green arrows); star shows blinking as horizontal black stripe; line represents cross-sectional scan of (b). (b) shows B-frame obtained in postprocessing through the neovascularisations seen in (a), with green arrows pointing at neovascularisations. The two horizontal lines are an artifact from the image processing.

\section{Acknowledgments}

This research was sponsored by the Gesellschaft der Freunde und Förderer der Augenklinik der LMU München e.V. as well as by the European Union projects FUN-OCT (FP7 HEALTH, Contract no. 201880) and FDML-Raman (FP7 ERC, Contract no. 259158).

\section{References}

[1] S. Resnikoff, D. Pascolini, D. Etya'ale et al., "Global data on visual impairment in the year 2002," Bulletin of the World Health Organization, vol. 82, no. 11, pp. 844-851, 2004.

[2] J. W. Y. Yau, S. L. Rogers, R. Kawasaki et al., "Global prevalence and major risk factors of diabetic retinopathy," Diabetes Care, vol. 35, no. 3, pp. 556-564, 2012.

[3] S. Ahsan, A. Basit, K. R. Ahmed et al., "Diagnostic accuracy of direct ophthalmoscopy for detection of diabetic retinopathy using fundus photographs as a reference standard," Diabetes \& Metabolic Syndrome, vol. 8, no. 2, pp. 96-101, 2014.

[4] S. Schwartz, M. Harasawa, V. Baldivieso, A. L. Sabel, N. Mandava, and H. Quiroz-Mercado, "Nonmydriatic fundus camera for diabetic retinopathy screening in a safety net hospital: effectiveness, prevalence, and risk factors," European Journal of Ophthalmology, vol. 25, no. 2, pp. 145-152, 2014.

[5] A. S. Neubauer, M. Kernt, C. Haritoglou, S. G. Priglinger, A. Kampik, and M. W. Ulbig, "Nonmydriatic screening for diabetic retinopathy by ultra-widefield scanning laser ophthalmoscopy (Optomap)," Graefe's Archive for Clinical and Experimental Ophthalmology, vol. 246, no. 2, pp. 229-235, 2008.

[6] M. G. Nittala, R. Konduru, H. Ruiz-Garcia, and S. R. Sadda, "Effect of OCT volume scan density on thickness measurements in diabetic macular edema," Eye, vol. 25, no. 10, pp. 1347-1355, 2011.

[7] H. J. Shin, S. H. Lee, H. Chung, and H. C. Kim, "Association between photoreceptor integrity and visual outcome in diabetic macular edema," Graefe's Archive for Clinical and Experimental Ophthalmology, vol. 250, no. 1, pp. 61-70, 2012.

[8] T. Hirano, Y. Iesato, Y. Toriyama, A. Imai, and T. Murata, "Detection of fovea-threatening diabetic macular edema by optical coherence tomography to maintain good vision by prophylactic treatment," Ophthalmic Research, vol. 52, no. 2, pp. 65-73, 2014.

[9] L. Reznicek, T. Klein, W. Wieser et al., "Megahertz ultra-widefield swept-source retina optical coherence tomography compared to current existing imaging devices," Graefe's Archive for Clinical and Experimental Ophthalmology, vol. 252, no. 6, pp. 1009-1016, 2014.

[10] T. Klein, W. Wieser, L. Reznicek, A. Neubauer, A. Kampik, and R. Huber, "Multi-MHz retinal OCT," Biomedical Optics Express, vol. 4, no. 10, pp. 1890-1908, 2013.

[11] W. Wieser, B. R. Biedermann, T. Klein, C. M. Eigenwillig, and R. Huber, "Multi-megahertz OCT: high quality 3D imaging at 20 million A-scans and 4.5 GVoxels per second," Optics Express, vol. 18, no. 14, pp. 14685-14704, 2010.

[12] T. Klein, W. Wieser, C. M. Eigenwillig, B. R. Biedermann, and R. Huber, "Megahertz OCT for ultrawide-field retinal imaging with a $1050 \mathrm{~nm}$ Fourier domain mode-locked laser," Optics Express, vol. 19, no. 4, pp. 3044-3062, 2011.

[13] R. Huber, D. C. Adler, V. J. Srinivasan, and J. G. Fujimoto, "Fourier domain mode locking at $1050 \mathrm{~nm}$ for ultra-high-speed optical coherence tomography of the human retina at 236,000 axial scans per second," Optics Letters, vol. 32, no. 14, pp. 2049-2051, 2007.

[14] M. Kernt, S. Cserhati, F. Seidensticker et al., "Improvement of diabetic retinopathy with intravitreal Ranibizumab," Diabetes Research and Clinical Practice, vol. 100, no. 1, pp. ell-e13, 2013. 


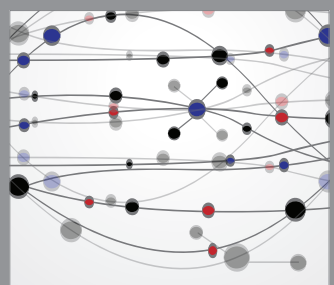

The Scientific World Journal
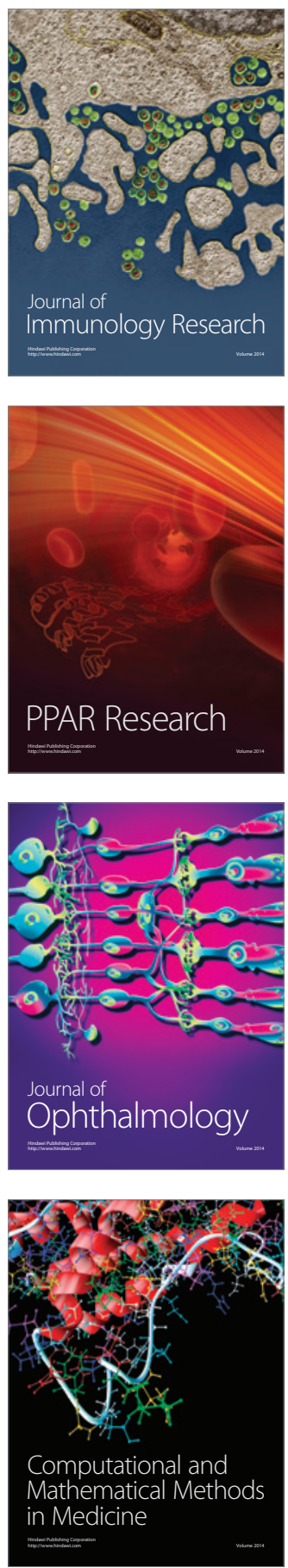

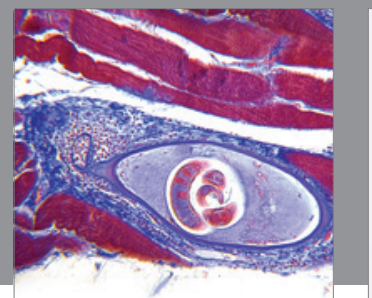

Gastroenterology

Research and Practice
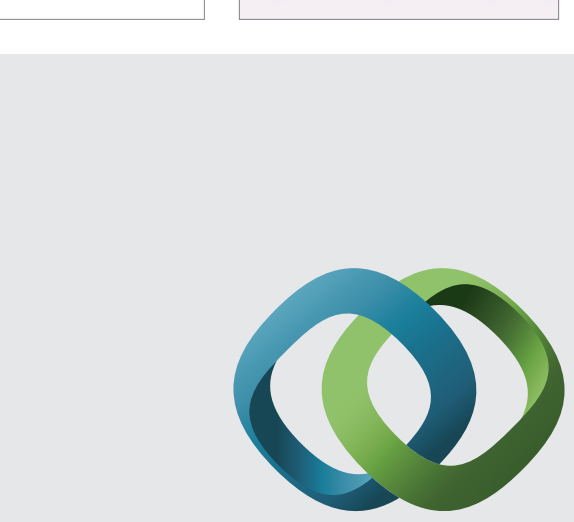

\section{Hindawi}

Submit your manuscripts at

http://www.hindawi.com
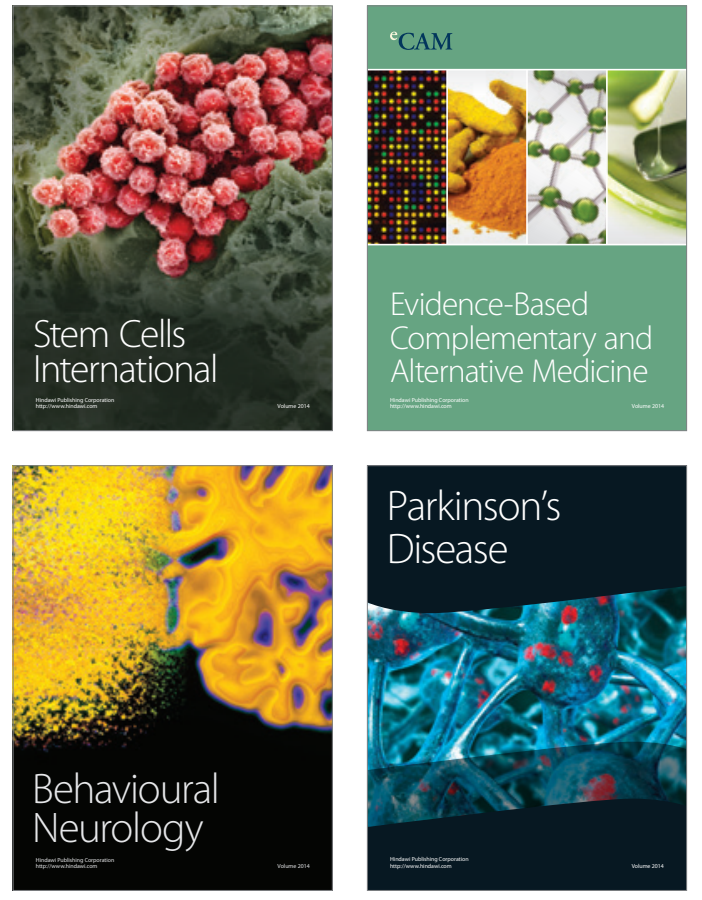
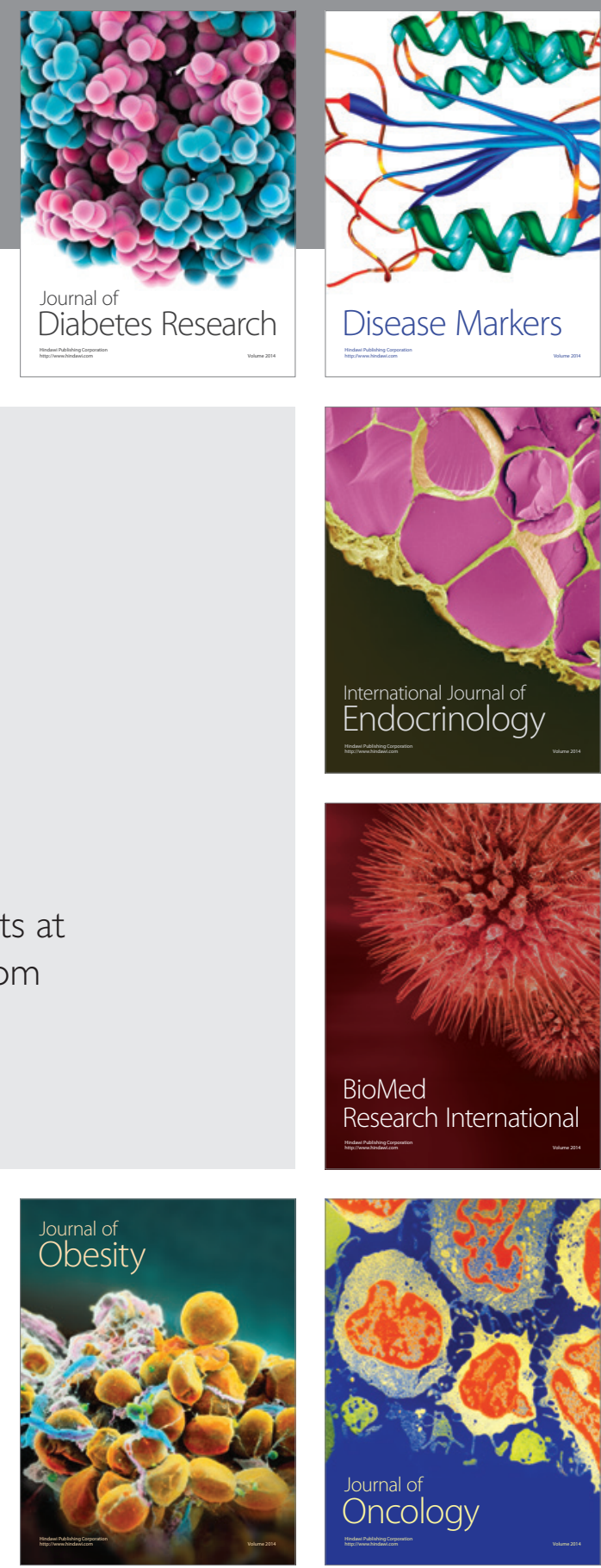

Disease Markers
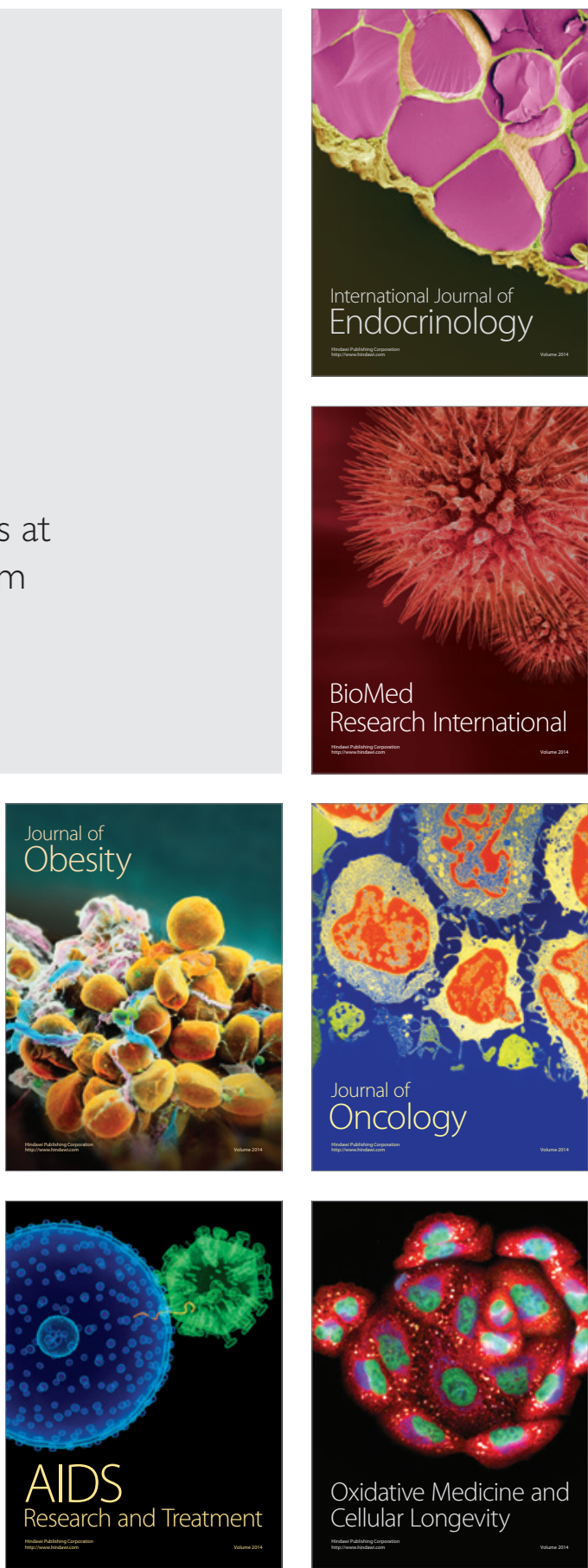